\title{
SOURCES AND PATHWAYS OF SPREAD OF VANCOMYCIN-RESISTANT ENTEROCOCCI IN HEMATO-ONCOLOGICAL PATIENTS
}

\author{
Iva Vagnerova ${ }^{\mathrm{a}}$, Pavel Sauer ${ }^{\mathrm{a}}$, Milan Kolara, Sabina Slepickova ${ }^{\mathrm{a}}$, Jaromir Hubacek ${ }^{\mathrm{b}}$, \\ Edgar Faber ${ }^{\mathrm{b}}$, Ludek Raida ${ }^{\mathrm{b}}$, Tomas Papajik \\ a Department of Microbiology, Faculty of Medicine, Palacky University, University Hospital Olomouc, Czech Republic \\ ${ }^{b}$ Department of Hemato-oncology, Faculty of Medicine, Palacky University, University Hospital Olomouc \\ e-mail:wagneri@fnol.cz
}

Received: April 21, 2006; Accepted: June 2, 2006

Key words: Vancomycin-resistant enterococci/Epidemiology

The presented study aims at analyzing an increasing prevalence of vancomycin-resistant enterococci (VRE) isolated from various kinds of clinical material obtained from patients in the Department of Hemato-oncology (DHO), University Hospital in Olomouc, Czech Republic.

Between January 1 and March 31, 2005, enterococci were isolated by standard microbiological procedures using both clinical material obtained from hospitalized patients and samples from the department environment. Resistance to vancomycin and teicoplanin was determined by a standardized microdilution method. Phenotype determination of resistance to vancomycin was verified by PCR detection of vanA and vanB genes. In VanA Enterococcus faecium, macrorestriction analysis was performed by pulsed-field gel electrophoresis.

During the monitored period, a total of 128 Enterococcus sp. strains were isolated, of which 38 ( $30 \%$ ) isolates from 22 different patients were determined as VRE. Dominating were Enterococcus faecium VanA (63\%) and Enterococcus casseliflavus VanC (16 \%) strains. At the same time, one Enterococcus faecium VanA strain was acquired from a bed-side table used by a patient in whom a similar strain had been isolated repeatedly from various clinical materials including a rectal swab taken in 2004. Based on the macrorestriction analysis of genome DNA in 24 vancomycin-resistant Enterococcus faecium VanA strains isolated from the patients' clinical material, one strain from the bed-side table surface and one strain isolated from stools in 2004, 8 unique restriction profiles with similarity ranging from $90 \%$ to $100 \%$ were identified, which could be classified into 3 clonal types.

Thus, we can assume not only the endogenous origin of the VRE in hemato-oncological patients and their potential selection caused by therapy with broad-spectrum antibiotics but also the ability of the strains to survive in a hospital setting and, subsequently, to be spread clonally by various vectors.

\section{INTRODUCTION}

Recently, the importance of gram-positive, often multiresistant, bacteria in the etiopathogenesis of - particularly nosocomial - infectious has increased. Among these, a significant role is played by vancomycin-resistant enterococci (VRE) present especially in patients with hematooncological diseases and those in intensive care units ${ }^{1}$. Although they mostly represent mere colonization of the gastrointestinal tract they can also cause serious infections which are difficult to manage therapeutically.

VRE were first isolated in Europe in 1986 and a year later in the USA ${ }^{2,3,4}$. At present, VRE are described in other countries in the world. In the Czech Republic, VRE were first reported at the Department of Hematooncology, University Hospital Olomouc (DHO) in $1997^{5}$.

The presented study aims at analysing an increased prevalence of VRE in the DHO patients in early 2005, assessing their importance and formulating a hypothesis concerning their source and potential transmission path- ways as based on a molecular biology analysis of VanA Enterococcus faecium strains.

\section{MATERIAL AND METHODS}

By using standard microbiological procedures, enterococci were isolated from clinical material (sputum and upper respiratory tract swabs, pus, blood, samples from cannula and catheter surfaces, urine and rectal swabs) obtained from patients hospitalized in the DHO between January 1 and March 31, 2005. Enterococcal strains were identified according to the criteria of Facklam and Collins and biochemical properties using the EN-COCCUStest (Pliva-Lachema) $)^{6}$.

At the same time, enterococci were isolated from the above mentioned department environment. Samples were taken with a moistened cotton pad from a $100 \times 100 \mathrm{~mm}$ area in two directions at right angles to each other. The samples were immediately inoculated into $5 \mathrm{ml}$ Nutrient 
Broth $\mathrm{w} / 1 \%$ peptone and $48 \mathrm{~h}$ cultivation at $37{ }^{\circ} \mathrm{C}$ was initiated. Following that, the liquid medium was inoculated with a calibrated loop on a solid medium (Columbia Blood Agar Base with 7 \% sheep blood) and incubated under aerobic conditions at $37^{\circ} \mathrm{C}$ for $48 \mathrm{~h}$.

Resistance to vancomycin and teicoplanin was determined by a standardized microdilution $\operatorname{method}^{7}$. The limit concentrations for susceptible strains were set at 4 $\mathrm{mg} / \mathrm{L}$ for vancomycin and $8 \mathrm{mg} / \mathrm{L}$ for teicoplanin. The dilution accuracy of both antibiotics was verified with Staphylococcus aureus ATCC 29213 and Enterococcus faecalis ATCC 29212 reference strains. Phenotype assessment of resistance to vancomycin was verified by PCR detection of $v a n A$ and $v a n B$ genes ${ }^{8}$.

In a particular patient, one strain of a given VRE species isolated from each type of clinical material as the first one was added to the database.

All VanA E. faecium strains were assessed by macrorestriction analysis using pulsed-field gel electrophoresis (PFGE). To determine strain relationship genome DNA fragments were compared which had been cleaved by SmaI restriction endonuclease (Roche Diagnostics) and separated by PFGE (CHEF Mapper, Bio-Rad). The PFGE profiles of individual isolates were evaluated according to the criteria described by Goering ${ }^{9}$. The results were statistically processed by a cluster analysis (Gel Compar, Applied Maths) as used by Kolar et al. for molecular biology analysis of VRE of animal origin ${ }^{10}$.

\section{RESULTS}

During the observed period, a total of 128 Enterococcus sp. strains were isolated from biological material obtained from the DHO patients. Of those, 38 (30 \%) isolates from 22 different patients were determined as VRE. Their species, phenotypes and genotypes are listed in Table 1. Dominant were VanA E. faecium (63\%) and VanC E. casseliflavus (16\%) strains. At the same time, one VanA Enterococcus faecium strain was acquired from the department environment, namely a bed-side table used by a patient in whom a similar strain had been isolated repeatedly from various clinical materials (oral cavity, urine, stools, and even stools analyzed in 2004).

Table 2 gives an overview of VRE in individual types of clinical material. Most frequently, VRE were isolated from rectal swabs ( $76 \%$ ), less frequently from urine $(21 \%)$ and only in $3 \%$ from the upper respiratory tract swabs. No VRE strain was detected in blood.

In the case of 5 (23\%) patients, VRE were isolated not only from stools but also from urine. One patient had VRE in stools, urine and the upper respiratory tract swab, all at once. Of particular interest is that in 5 patients 2 VRE species were detected (E. faecium + Enterococcus faecalis, E. faecium + E. casseliflavus, E. faecium + E. sp. $)$ and in one patient even 3 species ( $E$. faecium, E. faecalis, E. sp.). The most common isolate, VanA E. faecium (24 strains from 17 patients), was cultivated solely from stools in $11(64 \%)$ patients, both from stools and urine in 5 patients ( $30 \%$ ) and from stools, urine and the upper respiratory tract swab in one patient. Epidemiologically important is the fact that during the week of February

Table 1. Phenotypes and genotypes of VRE in clinical material from the DHO patients.

\begin{tabular}{|l|c|c|c|c|}
\hline \multirow{2}{*}{ Species } & \multirow{2}{*}{ Phenotype } & \multirow{2}{*}{ Genotype } & \multicolumn{2}{|c|}{ Prevalence } \\
\cline { 3 - 5 } & & & Number of strains & Percentage \\
\hline Enterococcus faecium & VanA & vanA & 24 & 63 \\
\hline Enterococcus faecalis & VanA & vanA & 3 & 8 \\
\hline Enterococcus faecalis & VanB & vanB & 1 & 3 \\
\hline Enterococcus casseliflavus & VanC & NT & 6 & 16 \\
\hline Enterococcus sp. & VanA & vanA & 4 & 10 \\
\hline
\end{tabular}

NT - not tested

Table 2. VRE in clinical material from the DHO patients.

\begin{tabular}{|l|c|c|}
\hline Material & Number of isolated VRE & Relative frequency (\%) \\
\hline Rectal swab & 29 & 76 \\
\hline Urine & 8 & 31 \\
\hline Upper respiratory tract swabs & 1 & 3 \\
\hline
\end{tabular}


21-28, 2005, the VanA E. faecium strains were newly demonstrated in 7 patients ( $32 \%$ of all patients, $41 \%$ of patients with VanA E. faecium).

The macrorestriction analysis of genome DNA in 24 vancomycin-resistant E. faecium VanA strains isolated from clinical material obtained from 17 patients, 1 strain from a bed-side table surface and 1 strain isolated from a patient in the group as early as in 2004 resulted in identification of 8 unique restriction profiles that could be classified into three clonal types. The clonal type A contained 15 strains which were 100 percent identical and detected between February 9 and March 26, 2005. Of those, 7 strains were isolated during a single week as stated above. Eleven strains were from stools, 2 from urine, 1 from the oral cavity and 1 from the environment - a surface of a bed-side table used by a patient with isolated VanA E. faecium. In one patient, the isolation was simultaneous from both stools and urine, in another patient from urine, the oral cavity and the bed-side table surface. Five strains isolated between January 12 and March 29, 2005 with concordance from $90 \%$ to $100 \%$ could be classified as type B. These were 2 strains from stools and 3 strains from urine, in one patient the same strains were isolated simultaneously from both stools and urine. The C clonal type comprised 4 strains with $99 \%$ concordance - 3 strains were from the patients' stools from the period of January 11 to March 10, 2005, and one strain was from the 2004 stools test in a patient in whom three other VRE of the clonal type A were isolated (from urine, the oral cavity and the bed-side table surface). The remaining two strains were of a unique restriction profile and could not be assigned to any of the above mentioned clonal types. The PFGE results showing relationship of all the 26 analyzed strains are in Fig. 1.

\section{DISCUSSION}

The presence of VRE poses a potential risk especially for hemato-oncological, oncological and other patients with significant immunosuppression. Most cases are those with mere colonization of the gastrointestinal tract. However, VRE strains may act as etiological agents of serious urinary and respiratory infections and generalized sepses ${ }^{11}$.

From an epidemiological point of view, the most dangerous are VanA and VanB phenotypes of VRE as they represent resistance which is acquired and transferable ${ }^{12, \text { "é13 }}$. Since 1997, VRE have been regularly isolated from clinical material obtained from the DHO patients and their frequency rate among all enterococcal isolates reaches $5 \%$ on average $e^{5,14}$.

Due to the increased prevalence of VRE in the first three months of 2005 (30\%) a macrorestriction analysis of genome DNA of VanA E. faecium strains isolated from clinical material obtained from the DHO patients and one strain isolated from the department environment was carried out. The results suggest both endogenous and exogenous sources of the strains. The endogenous origin can be proved by detection of 8 unique restriction profiles. It can be assumed that on their admission to the hospital some patients are already colonized with VRE

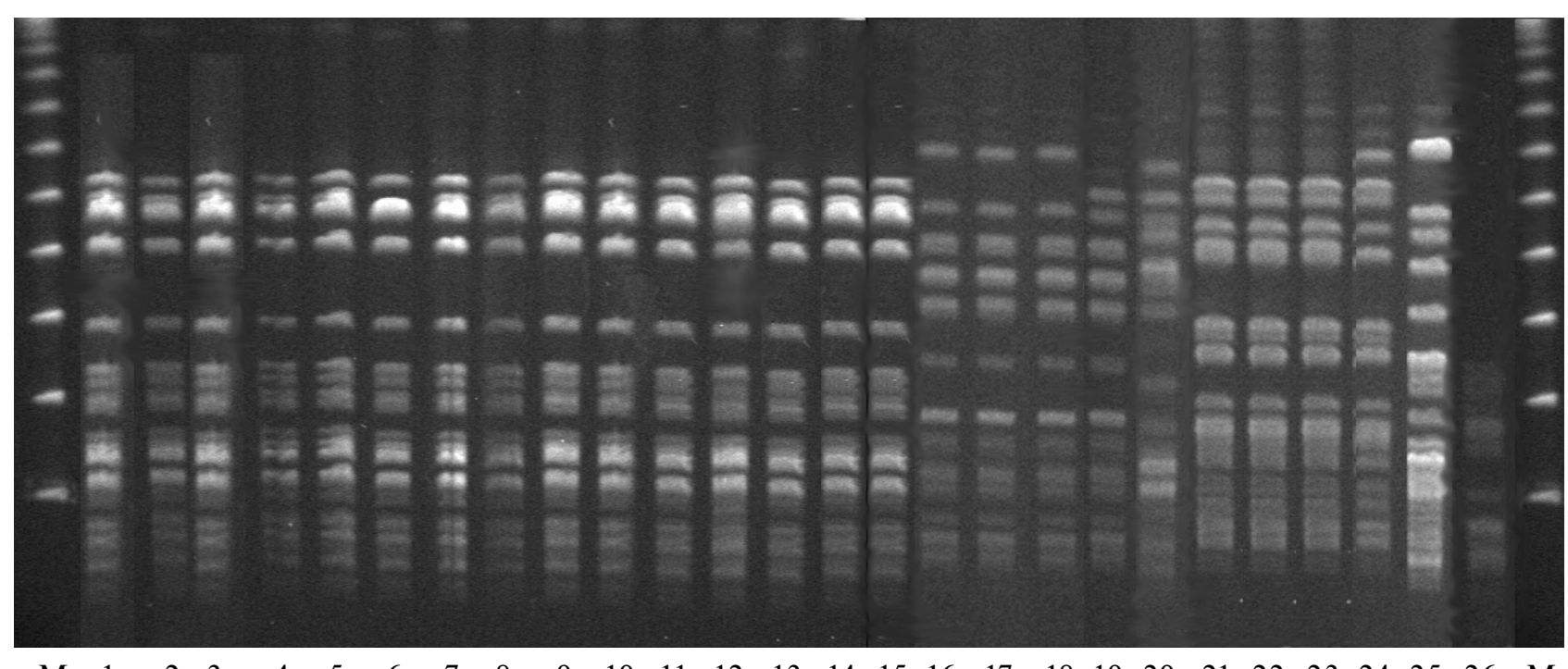

$\begin{array}{llllllllllllllllllllllllllll}\mathrm{M} & 1 & 2 & 3 & 4 & 5 & 6 & 7 & 8 & 9 & 10 & 11 & 12 & 13 & 14 & 15 & 16 & 17 & 18 & 19 & 20 & 21 & 22 & 23 & 24 & 25 & 26 & \mathrm{M}\end{array}$

Legend

Lanes M: molecular weight marker (Sigma); Lanes 1-15: isolates of restriction clonal type A; Lanes 16-20: isolates of restriction clonal type B; Lanes 21-24: isolates of restriction clonal type C; Lanes 25-26 isolates of unique restriction profile

Fig. 1. PFGE fingerprinting of E. faecium isolates after digestion with SmaI 
and their subsequent selection and multiplication results from radical therapy with broad-spectrum antimicrobial agents. Consequently, these strains may be transferred exogenously to other patients by various vectors - most commonly by health personnel's hands. Thus, their clonal spread occurs as demonstrated by the three clonal types present in our group.

VRE are markedly resistant bacteria which makes them capable of surviving both in the external environment and as a part of the gastrointestinal tract microflora, as documented by the isolation of a completely identical strain from a patient's stools in 2004 and stools of two other patients in 2005. The original assumption that the 2004 strain could have been the primary source for the clonal type A was not confirmed as the isolate belonged to the $\mathrm{C}$ clone and its carrier's stools contained a completely different strain with a unique restriction profile in 2005. Although other VRE strains of the particular patient obtained from urine, the oral cavity and the bed-side table surface belonged to the clonal type A they were not isolated as the first ones over the mentioned period. The most frequent VanA E. faecium strain of the clonal type A originated from another patient's stools.

The results of the presented study clearly confirm both an endogenous origin of VRE and their potential capability of clonal and horizontal spread in a hospital environment. It is obvious that, apart from the rational use of antimicrobial agents, the prevention of the spread of bacterial resistance, including VRE, can only be achieved by applying strict hygienic and epidemiological measures.

\section{ACKNOWLEDGEMENT}

Partial results were supported by the VZ MSM 6198959205 project and by the Internal Grant Agency of the Czech Ministry of Health projects no. NR/9065-3.

\section{REFERENCES}

1. Speller DCE, Lynn WA, Rogers TR. (1995) Glycopeptide resistance in gram-positive bacteria. Clin Microbiol Inf 1, 54-59.

2. Leclercq R, Derlot E, Duval J, Courvalin P. (1988) Plasmid-mediated resistance to vancomycin and teicoplanin in E. faecium. N Engl J Med 319, 157-161.

3. Utlley AHC, Collins CH, Naidoo J, George RC. (1988) Vancomycin-resistant enterococci. Lancet 1, 57-58.

4. Kaplan RR, Gilligan PH, Facklam RR. (1988) Recovery of resistant enterococci during vancomycin prophylaxis. J Clin Microbiol 26, 1216-1218.

5. Koláŕ M, Vágnerová I, Kohnová I. (1997) Záchyt vankomycinrezistentních enterokoků ve Fakultní nemocnici v Olomouci. Klin Mikrobiol Inf Lék 3, 189-191.

6. Facklam RR, Collins MD. (1989) Identification of Enterococcus species isolated from human infections by conventional test scheme. J Clin Microbiol 27, 731-734.

7. National Committee for Clinical Laboratory Standards. Performance standards for antimicrobial susceptibility testing. Twelfth informational supplement. NCCLS document M100-S12. NCCLS, Wayne, Pennsylvania, USA 2002.

8. Dutka-Malen S, Evers S, Courvalin P. (1995) Detection of glycopeptide resistance genotypes and identification to the species level of clinically relevant enterococci by PCR. J Clin Microbiol 33, 24-27.

9. Goering RV. The molecular epidemiology of nosocomial infection. In: Specter S, editor. Rapid detection of infectious agents. New York: Plenum Press, 1998. p. 131-157.

10. Kolář M, Pantůček R, Bardoň J, Vagnerova I, Typovska H, Valka I, Doskar J. (2002) Occurrence of antibiotic-resistant bacterial strains isolated in poultry. Vet Med Czech 47, 52-59.

11. Peta M, Carretto E, Barbarini D, Zamperoni A, Carnevale L, Perversi L, Pagani M, Bonora MG, Fontana R, Marone P, Langer M. (2006) Outbreak of vancomycin-resistant Enterococcus spp. in an Italian general intensive care unit. Clin Microbiol Infect 12, 163-169.

12. Murray BE. (1997) Vancomycin-resistant enterococci. Am J Med 101, 284-293.

13. Murray BE. (1998) Diversity among multidrug-resistant enterococci. Emerg Infect Dis 4, 37-47.

14. Kolar M, Pantucek R, Vagnerova I, Kesselova M, Sauer P, Matouskova I, Doskar J, Koukalova D, Hejnar P. (2006) Genotypic characterisation of vancomycin-resistant Enterococcus faecium isolates from haemato-oncological patients at Olomouc University Hospital, Czech Republic. Clin Microbiol Infect 12, 353-360. 